\title{
How Neutrino and Charged Fermion Masses Are Connected Within Minimal Supersymmetric SO(10)
}

\author{
Borut Bajc* \\ J. Stefan Institute, Ljubljana, Slovenia \\ E-mail: B̈orut.Bajc@ijs.sì

\section{Goran Senjanović ${ }^{\dagger}$} \\ International Centre for Theoretical Physics, Trieste, Italy \\ E-mail: 'goran@ictp.trieste.it'

\section{Francesco Vissani ${ }^{\ddagger}$} \\ INFN, Laboratori Nazionali del Gran Sasso, Italy \\ E-mail: 'vissani@lngs.infn.it'
}

Abstract: Massive neutrinos are a generic prediction of $\mathrm{SO}(10)$, and models of unification cry for supersymmetry. Since we have a rather detailed information on neutrino and charged fermion masses, the real question is: how/whether it is possible to build a $\mathrm{SO}(10)$ supersymmetric model, that correctly incorporates fermion masses. We show that a simple construction is possible in the context of a minimal theory. We concentrate on the two heaviest generations, discuss the predictions of the model, and briefly comment on open questions.

\section{Yukawa Couplings at $M_{\mathrm{GUT}}$}

In order to avoid unacceptably big Dirac neutrino masses in $\mathrm{SO}(10)$ [i], one introduces 126-plets scalars. These produce huge Majorana masses for $\nu^{c}[\overline{2}]$, and decouple them from the light spectrum:

$$
\mathcal{L}=-\mathbf{1 6}_{i}\left[Y_{i j}^{(10)} \underline{\mathbf{1 0}}+Y_{i j}^{(126)} \underline{\mathbf{1 2 6}}\right] \mathbf{1 6}_{j}+\text { h.c. }
$$

The 10-plet contains two Higgs doublets, that we call $\varphi_{u}$ and $\varphi_{d}$, while the 126-plet contains one singlet $S$ (needed for $\nu^{c}$ ), one triplet $\Delta$ (which may contribute to light neutrino masses $\left[\overline{B i}_{1}\right)$ and two doublets $\varphi_{u}^{\prime}$ and $\varphi_{d}^{\prime}$ (useful to make up for wrong $\operatorname{SO}(10)$ mass relations

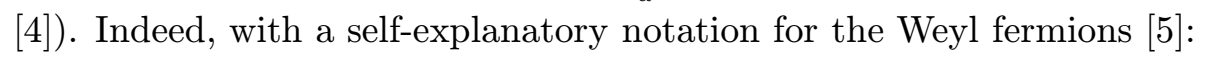

$$
\left\{\begin{array}{l}
\mathbf{1 6}_{i} \underline{\mathbf{1 0}} 16_{j} \ni \varphi_{u}\left(u_{i}^{c} u_{j}+\nu_{i}^{c} \nu_{j}\right)+\varphi_{d}\left(d_{i}^{c} d_{j}+\mathrm{e}_{i}^{c} \mathrm{e}_{j}\right)+(i \leftrightarrow j) ; \\
\mathbf{1 6}_{i} \underline{\mathbf{1 2 6}} 16_{j} \ni \frac{1}{2}\left(S \nu_{i}^{c} \nu_{j}^{c}+\Delta \nu_{i} \nu_{j}\right)+\varphi_{u}^{\prime}\left(u_{i}^{c} u_{j}-3 \nu_{i}^{c} \nu_{j}\right)+\varphi_{d}^{\prime}\left(d_{i}^{c} d_{j}-3 \mathrm{e}_{i}^{c} \mathrm{e}_{j}\right)+(i \leftrightarrow j)
\end{array}\right.
$$

${ }^{*}$ Supported by the Ministry of Education, Science and Sport of the Republic of Slovenia.

${ }^{\dagger}$ Partially supported by EEC under TMR contracts ERBFMRX-CT960090; HPRN-CT-2000-00152.

${ }^{\ddagger}$ Speaker. 

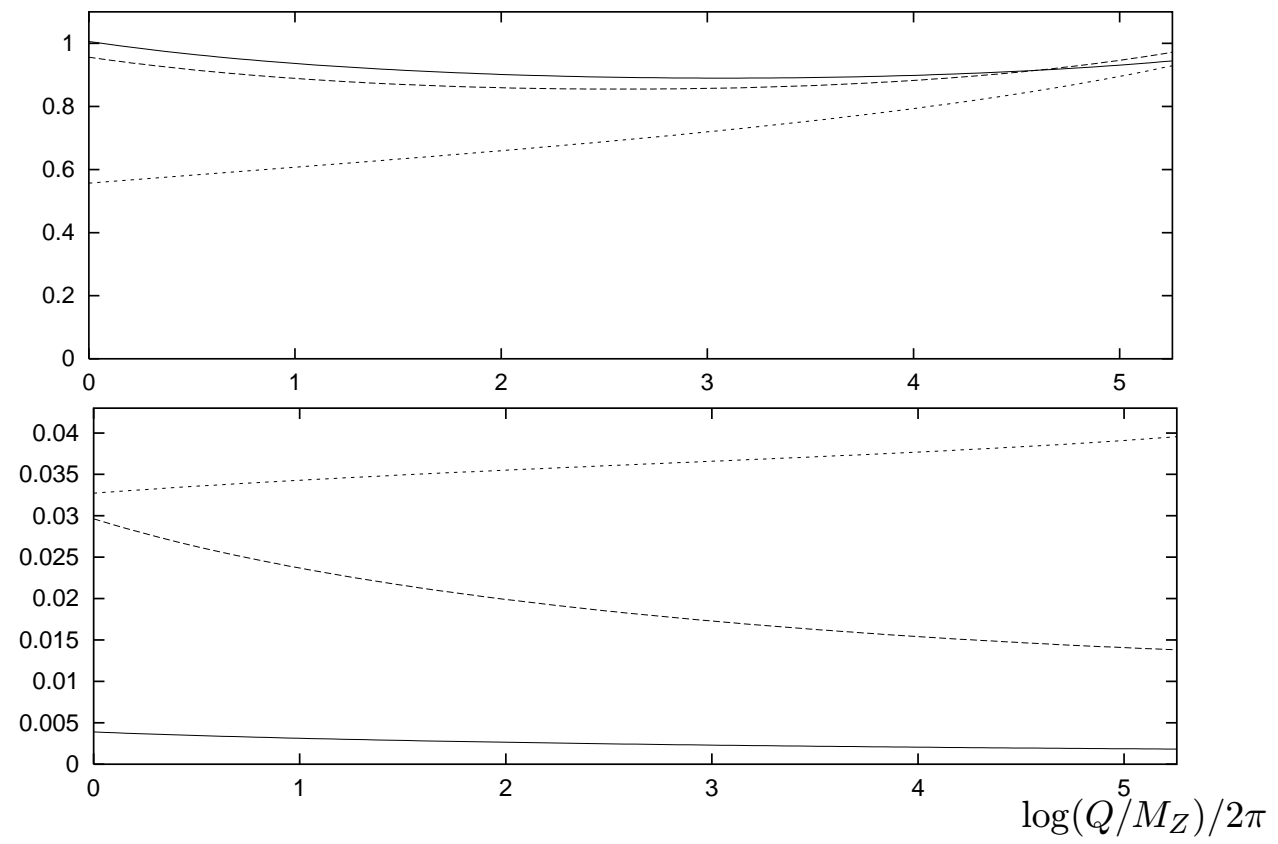

Figure 1: Upper panel: Running of MSSM Yukawa couplings of third generation from $M_{Z}$ till $M_{\mathrm{GUT}}$ ( $y_{t}$ is the largest at $M_{Z}, y_{\tau}$ the smallest). Lower panel: same for second generation $\left(y_{\mu}\right.$ is the largest, $y_{c}$ is the smallest). (We denote by $y_{x}$ the Yukawa coupling of the particle $x$, e.g.: $y_{t}$ for top, $y_{c}$ for charm, $y_{\mu}$ for muon. For a given $\tan \beta, y_{x}$ is computed from the mass of $x$ at $T=0$.)

\section{Beyond the Great (Supersymmetric) Desert}

The question of starting up model building is: what does the minimal supersymmetric standard model (MSSM) want from $\mathrm{SO}(10)$ ? We get an answer by extrapolating the Yukawa couplings from $T=0$ to $T=\log \left(M_{\mathrm{GUT}} / M_{Z}\right) / 2 \pi \approx 5.2$ (supersymmetry assumed, in order to comply with one-step unification of gauge couplings). From figure 1, one sees that:

- For $3^{\text {rd }}$ family charged fermions masses: the Hypothesis of leading $\underline{\mathbf{1 0}}$-plet Yukawa coupling [6]" that gives $y_{t}=y_{b}=y_{\tau}$ at $M_{\mathrm{GUT}}$ is $\mathrm{OK} .{ }^{1}$

- For $2^{\text {nd }}$ family charged fermion masses: the Hypothesis of leading $\underline{\mathbf{1 2 6}}$-plet Yukawa coupling [i]], that gives $y_{\mu}=-3 \times y_{s}$ at $M_{\mathrm{GUT}}$ is $\mathrm{OK}$.

This could be an accidental fact, but is suggestive enough to take it seriously.

\section{Determining Model and Parameters}

Now that we defined the target, the question becomes: how to match MSSM and SO(10) Yukawa couplings? SO(10) can meet the MSSM needs (illustrated in previous figure) after the very simple identification of the MSSM Higgs fields: $H_{u} \approx \varphi_{u}$ and $H_{d} \approx \varphi_{d}+\varepsilon \varphi_{d}^{\prime}$. (Of course, the orthogonal doublets should decouple from the MSSM spectrum, to maintain gauge coupling unification- namely, we need a "doublet-doublet" splitting).

\footnotetext{
${ }^{1}$ We tuned the vev ratio $\tan \beta=\left\langle H_{u}\right\rangle /\left\langle H_{d}\right\rangle \sim 55.4$ to get this. We use 1 loop "running" and $\alpha_{s}=0.118$.
} 
This position leads us to identify the MSSM Yukawa couplings in the following manner:

$$
\left\{\begin{array}{l}
Y_{u} \approx Y^{(10)} \quad \text { diagonal by definition } \\
Y_{d} \approx Y^{(10)}+\epsilon Y^{(126)} \\
Y_{e} \approx Y^{(10)}-3 \epsilon Y^{(126)}
\end{array}\right.
$$

Since we know the Yukawa couplings (after extrapolation at $M_{\mathrm{GUT}}$ ), we can deduce the size of several elements of the $\mathrm{SO}(10)$ Yukawa matrices. The chain of deduction we follow and the numerical values we obtain at $M_{\mathrm{GUT}}$ are shown in this table:

$$
\begin{array}{|cl|}
\hline y_{t}, y_{b}, y_{\tau} & \Rightarrow \quad Y_{33}^{(10)} \simeq 0.94 \gg \epsilon Y_{33}^{(126)} \\
y_{\mu}, y_{s} & \Rightarrow \epsilon Y_{22}^{(126)} \simeq 1.4 \times 10^{-2}>Y_{22}^{(10)} \\
y_{c} & \Rightarrow Y_{22}^{(10)} \simeq 1.8 \times 10^{-3} \\
V_{c b} & \Rightarrow \epsilon Y_{23}^{(126)} \simeq 2.7 \times 10^{-2}
\end{array}
$$

Two remarks are in order:

- We kept the deduction as simple as possible e.g. we did not perform detailed diagonalizations to get these numbers, which saves us from considering their phases. (However, we feel that it is fair to say that higher order effects, threshold and non-log corrections etc. could make a much more accurate treatment meaningless.)

- The only unknown element of the $2^{n d}-3^{r d}$ family blocks is $\epsilon Y_{33}^{(126)}$ (though one may reasonably guess that it is not too far from $\epsilon Y_{22}^{(126)}$ or $\left.\epsilon Y_{23}^{(126)}\right)$.

\section{Neutrino Features}

It is time to pass to the fermion of the day, the neutrino. In order to formulate our proposal, we will base our discussion on this provocative question: what do these neutrinos want? We recapitulate the experimental situation by means of the following table:

\begin{tabular}{|clr|}
\hline$\Delta m_{31}^{2}$ & {$[1.5,5] \times 10^{-3} \mathrm{eV}^{2}$} & atmospheric neutrinos \\
$\Delta m_{21}^{2}$ & {$[2,50] \times 10^{-5} \mathrm{eV}^{2}$} & solar $\mathrm{LMA}$ (or $\left.<2 \times 10^{-7} \mathrm{eV}^{2}\right)$ \\
$\theta_{23}$ & {$\left[35^{\circ}, 55^{\circ}\right]$} & atmospheric neutrinos \\
$\theta_{13}$ & $<10^{\circ}$ & $\mathrm{CHOOZ}+$ atm. $+\mathrm{K} 2 \mathrm{~K}$ (depends on $\Delta m_{31}^{2}$ ) \\
$\theta_{12}$ & {$\left[25^{\circ}, 43^{\circ}\right]$} & solar neutrinos $(99 \% \mathrm{CL})$ \\
\hline
\end{tabular}

We will be mostly concerned with the first three items. As remarked by several people (see

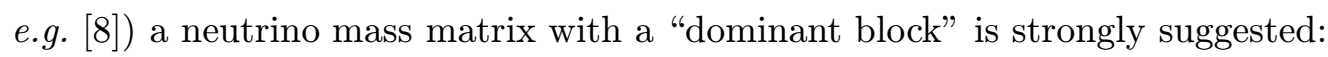

$$
\frac{\mathbf{M}_{\nu}}{\sqrt{\Delta m_{31}^{2}}}=\frac{1}{2} \times\left(\begin{array}{lll}
0 & 0 & 0 \\
0 & 1 & 1 \\
0 & 1 & 1
\end{array}\right)+\mathcal{O}\left(\theta_{13}, \theta_{23}-\frac{\pi}{4}, \sqrt{\frac{\Delta m_{21}^{2}}{\Delta m_{31}^{2}}}\right)
$$

But, due to hierarchical Yukawa couplings, the seesaw does not yield this pattern generically

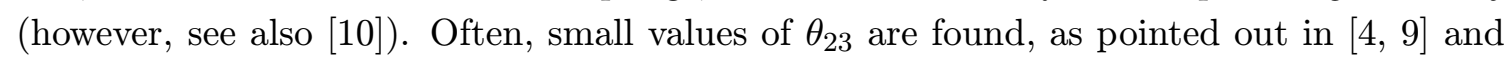
as illustrated here:

$$
M_{D} M_{R}^{-1} M_{D}=\left(\begin{array}{ll}
\epsilon & 0 \\
0 & 1
\end{array}\right) \cdot\left(\begin{array}{ll}
a & b \\
b & c
\end{array}\right) \cdot\left(\begin{array}{ll}
\epsilon & 0 \\
0 & 1
\end{array}\right)
$$




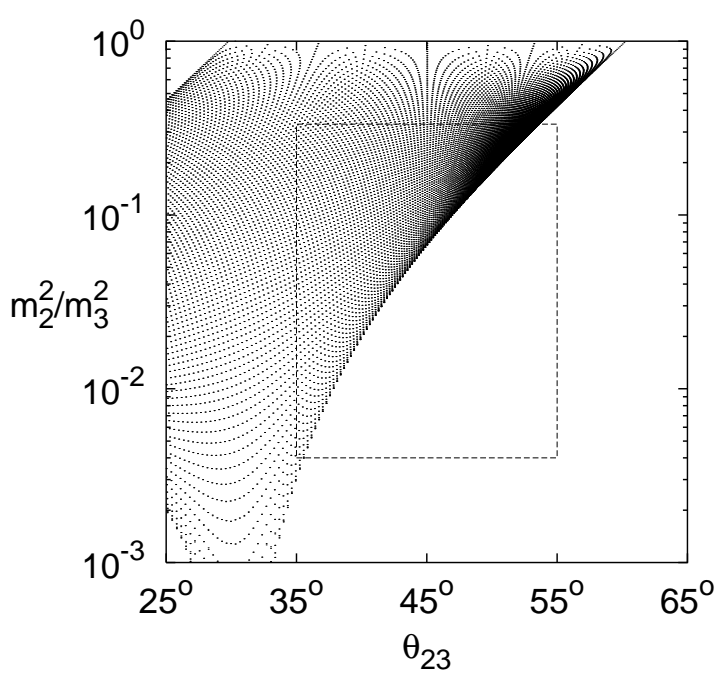

Figure 2: Possible values of the mass hierarchy parameter $m_{2}^{2} / m_{3}^{2}$ and of the atmospheric mixing angle $\theta_{23}$, obtained varying the complex input parameter $x$ (eq. 5.11 .1 . A rectangle encloses the range of permitted values, estimated assuming that the lightest neutrino mass $m_{1}$ is negligible.

Thus, we are lead to try another mass mechanism, and we welcome the fact that we have the triplet $\Delta$ at our disposal [3in] (by the way, we arrived at a common sense answer to the question on "neutrino wishes": neutrinos want to be different from the other fermions).

\section{The Triplet Option}

We are assuming that neutrinos take mass mostly from the triplet $\Delta: \mathbf{M}_{\nu} \propto Y^{(126)}$. Running back to $M_{Z}$ the MSSM Yukawa couplings, we get a simple expression for the $\nu_{\mu}-\nu_{\tau}$ block of the neutrino mass matrix:

$$
\mathbf{M}_{\nu} \propto\left(\begin{array}{cc}
1 & 1.7 \\
1.7 & x
\end{array}\right)
$$

(We have " $x$ ", for $Y_{33}^{(126)}$ is unknown, and also because seesaw might contribute to 33-

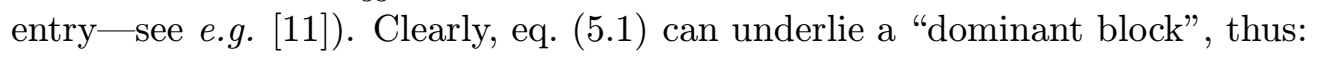

$$
\theta_{23} \text { can be large }
$$

we expect a weak mass hierarchy (not $m_{3} \gg m_{2}$ )

These two properties correlate, as can be seen in figure 2. To further illustrate this result (assuming $m_{3}^{2} \simeq \Delta m_{31}^{2}=3 \times 10^{-3} \mathrm{eV}^{2}$ and $m_{2}^{2} \simeq \Delta m_{21}^{2}$ ) we note that:

- If $\theta_{23}=45^{\circ}$, then $\Delta m_{21}^{2}>2 \times 10^{-4} \mathrm{eV}^{2}$;

- If $\Delta m_{21}^{2}=5 \times 10^{-5} \mathrm{eV}^{2}$, then ${ }^{2} \theta_{23}<40^{\circ}$.

\footnotetext{
${ }^{2}$ Quite tough to test experimentally, since it is equivalent to $\sin ^{2} 2 \theta_{23}<0.97 \ldots$
} 


\section{Summary and Discussion}

* We discussed an "economical embedding" of MSSM into SO(10), in a sense that all features of 126-plet have been exploited, namely: we use singlet, doublets and triplet vev's.

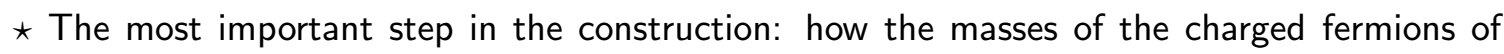
the $2^{\text {nd }}$ and $3^{\text {rd }}$ generations are explained (Sects. 2 and 3 ). $3^{\text {rd }}$ family unification suggests the large $\tan \beta=\left\langle H_{u}\right\rangle /\left\langle H_{d}\right\rangle$ regime; this is not an appealing case, but perhaps it is still viable (incidentally, it permits us to accommodate a "heavy" Higgs field, $m_{h}<135 \mathrm{GeV}$ ).

$\star$ The triplet mechanism for neutrino mass generation is at least likely (discussion in Sect. 4). The correlations among $\left(\mathbf{M}_{\nu}\right)_{22} \leftrightarrow m_{\mu}, m_{s}$, and $\left(\mathbf{M}_{\nu}\right)_{23} \leftrightarrow V_{c b}$ imply (eq. (…'1)):

$$
\theta_{23} \in\left[35^{\circ}, 55^{\circ}\right] \Leftrightarrow \frac{m_{2}^{2}}{m_{3}^{2}} \in\left[\frac{1}{250}, \frac{1}{3}\right]
$$

Solar $\nu$ solutions with big hierarchy are disfavored, while LMA fits well the scheme. After the $\Delta m_{21}^{2}$ measurement-at KamLAND?-we will get an upper bound on $\theta_{23}$ (fig. 2 and Sect. 5).

$\star$ A pending question is: masses of $1^{\text {st }}$ family fermions (also $m_{1}$ ); proton decay rate; feasibility of baryogenesis-through-leptogenesis mechanism. These features are strictly tied among them, and require further study.

To conclude, we stress the main goals achieved: We showed that it is possible to build a simple model for fermion masses based on supersymmetric $\mathrm{SO}(10)$, with renormalizable couplings only. This model accounts for the masses of second and third generation fermions. It has large $\theta_{23}$, and prefers the solar neutrino solutions with weak mass hierarchy.

\section{References}

[1] H. Georgi, in Coral Gables 1975, "Theories and experiments in high energy physics", New York 1975, 329-339; H. Fritzsch and P. Minkowski, Annals Phys. 93, 193 (1975).

[2] M. Gell-Mann et al. in "Supergravity", Stony Brook 1979; T. Yanagida in "Proceeding of the workshop on unified theory and baryon number in the Universe", KEK 1979;

R.N. Mohapatra and G. Senjanović, Phys. Rev. Lett. 44 (1980) 912.

[3] R.N. Mohapatra and G. Senjanović, Phys. Rev. D23, 165 (1981).

[4] K.S. Babu and R.N. Mohapatra, Phys. Rev. Lett. 70 (1993) 2845

[5] R. Barbieri, D.V. Nanopoulos, G. Morchio and F. Strocchi, Phys. Lett. B 90 (1980) 91.

[6] H. Georgi and D.V. Nanopoulos, Nucl. Phys. B 159 (1979) 16.

[7] H. Georgi and C. Jarlskog, Phys. Lett. B 86 (1979) 297.

[8] This remark was first done in Z.G. Berezhiani and A. Rossi, Phys. Lett. B 367 (1996) 219. A recent discussion in F. Vissani, $9^{\text {th }}$ M. Grossmann meeting, Rome 2000, hep-ph/0102235.

[9] B. Brahmachari and R.N. Mohapatra, Phys. Rev. D 58 (1998) 015001.

[10] There is not a "no-go theorem" for seesaw mechanism in $\mathrm{SO}(10)$. See K.T. Mahanthappa et al., hep-ph/0110037, talk given in the session on CP violation of HEP2001, and K. Matsuda et al., hep-ph/0108202, appeared after the conference. However, note that they find solutions with pronounced hierarchy as LOW and QVO, that are not expected in our model.

[11] As formalized in eq. (17) of A.S. Joshipura and E.A. Paschos, hep-ph/9906498. 